\title{
Alternative polyadenylation trans-factor FIP1 exacerbates UUO/RR-induced kidney injury and contributes to AKI-CKD transition via ROS-NLRP3 axis
}

Tong Zheng ${ }^{1}$, Yuqin Tan $\mathbb{D}^{1}$, Jiang $\mathrm{Qiu}^{2}$, Zhenwei Xie ${ }^{1}$, Xiao $\mathrm{Hu}^{1}$, Jinhua Zhang ${ }^{1}$ and Ning $\mathrm{Na}^{1}$

\begin{abstract}
NLRP3, a decisive role in inflammation regulation, is obviously upregulated by oxidative stress in kidney injury. The NLRP3 upregulation leads to unsolved inflammation and other pathological effects, contributing to aggravation of kidney injury and even transition to chronic kidney disease (CKD). However, the mechanism for NLRP3 upregulation and further aggravation of kidney injury remains largely elusive. In this study, we found NLRP3 $3^{\prime}$ UTR was shortened in response to kidney injury in vivo and oxidative stress in vitro. Functionally, such NLRP3 $3^{\prime} U T R$ shortening upregulated NLRP3 expression and amplified inflammation, fibrogenesis, ROS production and apoptosis, depending on stabilizing NLRP3 mRNA. Mechanistically, FIP1 was found to bind to PPAS of NLRP3 mRNA via its arginine-rich domain and to induce NLRP3 3'UTR shortening. In addition, FIP1 was upregulated in CKD specimens and negatively associated with renal function of CKD patients. More importantly, we found FIP1 was upregulated by oxidative stress and required for oxidative stress-induced NLRP3 upregulation, inflammation activation, cell damage and apoptosis. Finally, we proved that FIP1 silencing attenuated the inflammation activation, fibrogenesis, ROS production and apoptosis induced by UUO or IRI. Taken together, our results demonstrated that oxidative stress-upregulated FIP1 amplified inflammation, fibrogenesis, ROS production and apoptosis via inducing 3'UTR shortening of NLRP3, highlighting the importance of crosstalk between oxidative stress and alternative polyadenylation in AKI-CKD transition, as well as the therapeutic potential of FIP1 in kidney injury treatment.
\end{abstract}

\section{Introduction}

Acute and chronic kidney injury, generally caused by ischemia, obstructive pathologies and chemical drug, have become a major health concern worldwide with high mortality and morbidity ${ }^{1}$. The long-term ending of kidney injury is chronic kidney disease (CKD) and even kidney failure. Unfortunately, there remain no effective drugs for kidney injury ${ }^{2,3}$.

\footnotetext{
Correspondence: Yuqin Tan (ririan980522@126.com) or Ning Na (naning@mail.sysu.edu.cn)

'Department of Kidney Transplantation, The Third Affiliated Hospital of Sun Yat-sen University, Guangzhou, Guangdong, China

${ }^{2}$ Department of Organ Transplantation, The First Affiliated Hospital of Sun Yatsen University, Guangzhou, Guangdong, China

Edited by $Y$. Wang
}

In kidney injury, oxidative stress and inflammation are the most significant characteristics, as well as pivotal pathological factors ${ }^{4}$. In response to injury, inflammation initially emerges as a reno-protective role. However, amplified inflammation, especially induced by oxidative stress, turns into pro-injury effects in multiple ways ${ }^{5,6}$, making inhibition of amplified inflammation as a promising therapeutic strategy of kidney injury ${ }^{7-9}$.

NLRP3, the master component of inflammasome, is the central driver of inflammation ${ }^{10}$. Moreover, aberrant NLRP3 upregulation leads to overactivated inflammation and even $\mathrm{CKD}^{11,12}$. Although some biological events, especially the generation of reactive oxygen species (ROS), have been found to account for NLRP3 activation, the

\section{(c) The Author(s) 2021}

(c) (i) Open Access This article is licensed under a Creative Commons Attribution 4.0 International License, which permits use, sharing, adaptation, distribution and reproduction in any medium or format, as long as you give appropriate credit to the original author(s) and the source, provide a link to the Creative Commons license, and indicate if changes were made. The images or other third party material in this article are included in the article's Creative Commons license, unless indicated otherwise in a credit line to the material. If material is not included in the article's Creative Commons license and your intended use is not permitted by statutory regulation or exceeds the permitted use, you will need to obtain permission directly from the copyright holder. To view a copy of this license, visit http://creativecommons.org/licenses/by/4.0/. 
exact mechanism for disease-causing NLRP3 overactivation is still obscure ${ }^{13}$. On the other hand, none of NLRP3 inhibitors which have been proved effective in animal experiments is approved clinically, at least partly due to broad immunosuppressive side effects ${ }^{14}$. Therefore, to uncover the mechanism of NLRP3 overactivation is in great need, for improving NLRP3-targeted therapy.

Recently, the length change of mRNA $3^{\prime}$ untranslated regions $\left(3^{\prime} \mathrm{UTR}\right)$, derived from alternative polyadenylation (APA), has been identified as an emerging mechanism of oncogene overactivation ${ }^{15}$. Functionally, APA determines mRNA stability and translational efficiency mainly via regulating the response of mRNA to microRNA-mediated degradation, thus making a difference in final protein expression ${ }^{16,17}$. Considering microRNA's indispensable role in maintaining the homeostasis of NLRP3 expression ${ }^{18-20}$, APA may be an unrecognized contributor to NLRP3 overactivation, possibly offering better strategy for NLRP3-targeted therapy and even kidney injury treatment.

The goal of this study is to better understand the mechanism of inflammation overactivation upon kidney injury and whether APA participates in this process. Here we showed 3'UTR of NLRP3 mRNA is distinctly shortened in response to ischemia-reperfusion injury (IRI)/ unilateral ureteral obstruction (UUO) in vivo and to oxidative stress in vitro. Such $3^{\prime}$ UTR shortening amplifies inflammation, fibrogenesis, ROS production and apoptosis in kidney tubular epithelial cells via enhancing mRNA stability and protein expression of NLRP3. Mechanistically, we found the APA trans-factor FIP1 binds to proximal PAS of NLRP3 $3^{\prime} \mathrm{UTR}$ via its arginine-rich domain and induces NLRP3 3'UTR shortening. Moreover, obvious FIP1 upregulation is observed in both UUO/IRI kidney injury models and clinical CKD specimens. In particular, we demonstrated that oxidative stress induces such FIP1 upregulation and subsequent $3^{\prime}$ UTR shortening of NLRP3. Finally, we confirmed FIP1 knockdown alleviates the inflammation, fibrogenesis, ROS production and apoptosis induced by UUO or IRI. Thus, APA regulation is a key mechanism of NLRP3 overactivation and unsolved inflammation in kidney injury. To inhibit $3^{\prime}$ UTR shortening-induced NLRP3 overactivation via suppressing FIP1, is a promising strategy for NLRP3-targeted therapy and kidney injury treatment.

\section{Material and methods}

\section{Cell culture and hypoxia/reoxygenation (H/R) stimulation}

Tubular epithelial cell line HK-2 was a kind gift from Dr. Liutao Chen (Sun Yat-sen University). Tubular epithelial cell line HKC was purchase from cell bank of Chinese Academy of Medical Sciences. Cells were grown in DMEM/F-12 medium containing $10 \%$ fetal bovine serum (FBS) and incubated at $37^{\circ} \mathrm{C}$ in a humidified atmosphere with $5 \% \mathrm{CO}_{2}$. To induce $\mathrm{H} / \mathrm{R}$ model, cells were cultured in hypoxia $\left(1 \% \mathrm{O}_{2}, 94 \% \mathrm{~N}_{2}\right.$, and $\left.5 \% \mathrm{CO}_{2}\right)$ for 12 hours. And then, cells were cultured in normoxia (5\% $\mathrm{CO}_{2}$ and $95 \%$ air) for 6 hours. The control cells were incubated in normoxia ( $5 \% \mathrm{CO}_{2}$ and $95 \%$ air). Cells were routinely tested for Mycoplasma contamination.

\section{Western blot}

Immunoblot assays were performed according to standard protocol. Target proteins were detected with antibodies against NLRP3 (A12694, Abclonal, Wuhan, China), collagen I (A5786, Abclonal, Wuhan, China), fibronectin (A16678, Abclonal, Wuhan, China), Flag (F1804, Sigma, St Louis, USA), caspase1 (A0964, Abclonal, Wuhan, China), IL-1 $\beta$ (A11369, Abclonal, Wuhan, China), IL-18 (A1115, Abclonal, Wuhan, China), phossmad3 (AP0727, Abclonal, Wuhan, China), E-cadherin (3195, CST, Danvers, USA), N-cadherin (14215, CST, Danvers, USA), FIP1 (A7138, Abclonal, Wuhan, China), $\beta$-actin (AC026, Abclonal, Wuhan, China).

\section{Tissue specimens and immunohistochemical (IHC) staining}

The clinical tissue specimens of renal fibrosis were obtained from The First Affiliated Hospital of Sun Yat-sen (Guangzhou, China). IHC staining was performed as previously described ${ }^{21}$. Target proteins were detected with antibodies against NLRP3 (Servicebio, Wuhan, China), FIP1 (Abclonal, Wuhan, China), collagen I (Sevicebio, Wuhan, China), Fibronectin (Servicebio, Wuhan, China), CD3 (Servicebio, Wuhan, China) and F4/80 (Servicebio, Wuhan, China) and evaluated by two independent examiners who were blinded to the animal groups.

\section{qRT-PCR and the quantification of usage of distal PAS}

Total RNA was extracted from cells with Trizol reagent (Invitrogen, Carlsbad, USA) and reversely transcribed into cDNA with SuperScript III Reverse Transcriptase Kit (Invtriogen). The qRT-PCR assays were performed with SYBR-GREEN (Roche, Basel, Switzerland). A method which was previously described ${ }^{22,23}$ was used to quantify the usage of distal PAS. A pair of primers targeting the ORF was designed to represent the total transcripts, and another pair of primers targeting sequences just before the distal PAS (dPAS) to represent the long transcripts. The percentage of dPAS usage was calculated as follows: $\Delta \mathrm{Ct}_{\text {total }}\left(\Delta \mathrm{Ct}_{\text {distal }}\right)=\mathrm{Ct}_{\text {total }}\left(\mathrm{Ct}_{\text {distal }}\right)-\mathrm{Ct}_{\mathrm{GAPDH}} \cdot \Delta \Delta \mathrm{Ct}=$ $\Delta C \mathrm{t}_{\text {distal }}-\Delta \mathrm{Ct}_{\text {total }}$. Data are presented as the fold change normalized to the control, calculated as follows: $\Delta \Delta \Delta \mathrm{Ct}$

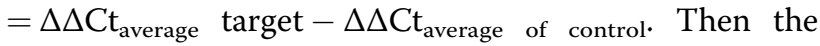
increase or decrease in dPAS usage was calculated as $\pm 2^{\text {-normalized } \Delta \Delta \Delta C \mathrm{t}}$. The negative value indicated that the mRNA had $3^{\prime}$ UTR shortening compared with the control. All primer sequences are listed in Supplementary Table 1. 


\section{Rapid amplification of $3^{\prime} \mathrm{CDNA}$ ends ( $3^{\prime}$ RACE) assay}

The NLRP3 cDNA was generated with $3^{\prime}$ Full Race Core Set (Takara, Kyoto, Japan). The first round PCR was performed with NLRP3 GSP-1 primer and 3'RACE outer primer. The nested PCR was performed with NLRP3 GSP2 primer and $3^{\prime} \mathrm{RACE}$ inner primer. The products of PCR were cloned with TA/Blunt-Zero cloning kit (Vazyme, Wuhan, China) and further sequenced. All primer sequences are listed in Supplementary Table 1.

\section{Luciferase reporter assay}

The short-3'UTR and long-3'UTR of NLRP3 were cloned downstream of the Renilla luciferase in psiCHECK-2. After transfection for 48 hours, luciferase activity was tested using the Dual-Luciferase Reporter Assay Kit (Promega, Madison, USA).

\section{Unilateral ureteral obstruction (UUO) and ischemia- reperfusion injury (IRI) kidney injury model}

8-week-old C57/BL6 male mice were used and randomly divided into groups as needed for kidney injury animal model. For UUO model, mice were anesthetized with pentobarbital sodium $(50 \mathrm{mg} / \mathrm{kg})$ and placed on a homeothermic table to maintain body temperature at $37^{\circ} \mathrm{C}$. Midline incision was made to open the abdomen of mice. Left ureter was ligated at proximal and distal points, and the ureter between ligated points was cut off. The abdomen was closed in layers. 14 days later, mice were sacrificed and the kidneys were collected. For IRI model, briefly, after a right nephrectomy, the left kidney was subjected to ischemia for 30 minutes with a nontraumatic vascular clamp, follow by 48 -hour reperfusion. Sham control animals were subjected to the identical operation without ischemia.

The serum or plasma was collected and stored at $-80{ }^{\circ} \mathrm{C}$ for further analysis. The kidney tissues were fixed in $4 \%$ paraformaldehyde (PFA) for histology analysis. The remaining kidney tissue was stored at $-80^{\circ} \mathrm{C}$ for biochemical analysis.

\section{Measurement of ROS production}

The ROS generation in cells and kidney tissues was detected with fluorescent probes DHE (KEYGEN Bio, Nanjing, China) and DCFH-DA (KEYGEN Bio, Nanjing, China). The cells or frozen specimens were incubated with DHE or DCFH-DA for 30 minutes. The fluorescent signal was captured with NIS elements and quantified with Image-pro Plus 6.0.

\section{Measurement of oxidative stress}

The commercial kits were purchased from Nanjing Jiancheng Bio (Nanjing, China) to measure oxidative stress in vivo and in vitro. The activity of superoxide dismutase (SOD, A001-3, hydroxylamine method) and concentration of malondialdehyde (MDA, A003-1, thiobarbituric acid method) were measured according to manufacturer's instruction.

\section{TUNEL assay}

The apoptosis in cells and kidney tissues was examined with commercial kit (Vazyme, Wuhan, China). Briefly, the samples were fixed and then exposed to the TUNEL reaction mixture containing TM red-labeled dUTP. After that, samples were counterstained with DAPI. TUNEL-positive nuclei were identified with fluorescence microscopy.

\section{RNA immunoprecipitation (RIP)}

$10^{8} \mathrm{HK} 2$ cells were used to extract total proteins with lysis buffer ( $40 \mathrm{mM}$ Tris, $120 \mathrm{mM} \mathrm{NaCl}, 1 \%$ Triton X-100, $1 \mathrm{mM} \mathrm{NaF}, 1 \mathrm{mM} \mathrm{Na} \mathrm{VO}_{4}, 1 \times$ protease inhibitor cocktail and $1 \mathrm{U} / \mathrm{ml}$ RNasin inhibitor). Protein A/G beads were incubated with Flag antibody and then used to isolate FIP1 protein, along with the FIP1-binding RNAs. TRIzol reagent was used to extract the co-precipitated RNAs. The amount of NLRP3 mRNA was analyzed by qRT-PCR.

\section{AAV9-mediated Fip1 knockdown in mice}

The plasmids pAAV2/9, pHelper and pAAV-ZsGreen1shRNA were obtained from Youbio company (Changsha, China). A Fip1 shRNA oligo was cloned into a pAAVZsGreen1-shRNA vector. These plasmids were transfected into HEK293 to develop the AAV9 expressing Fip1 shRNA or control shRNA. Then mice were anesthetized and $100 \mu$ AAV9 $\left(1 \times 10^{11}\right.$ viral genome particles $)$ was injected slowly into the renal vein using a $31 \mathrm{G}$ needle ${ }^{24}$. The shRNA sequences were listed as follows:Fip1 shRNA sense $\left(5^{\prime}-3^{\prime}\right)$ : GATCCCACTGAAGTAGACAACAATT CTCGAGAATTGTTGTCTACTTCAGTGGTTTTTG

Control shRNA sense $\left(5^{\prime}-3^{\prime}\right)$ : GATCCTTCTCCGAAC GTGTCACGTCTCGAGACGTGACACGTTCGGAGAA TTTTTG

\section{Statistical analysis}

In each experiment, data were presented as the mean \pm S.E.M. Student's t test was used to analysis the statistical significance between groups. The person correlation test was used to analysis the correlation. Data were performed with GraphPad Prism 8.0.

\section{Results}

$3^{\prime}$ UTR of NLRP3 is shortened in response to kidney injury

To investigate whether APA participates in NLRP3 overactivation upon kidney injury, UUO and IRI kidney injury models were used. The degree of difference of $3^{\prime}$ UTR usage of NLRP3 in five pairs of UUO-induced injured kidneys and matched unobstructed kidneys was examined by qRT-PCR. Compared with contralateral kidneys, short 3'UTR isoform of NLRP3 was obviously 
A $\square$ Uuo

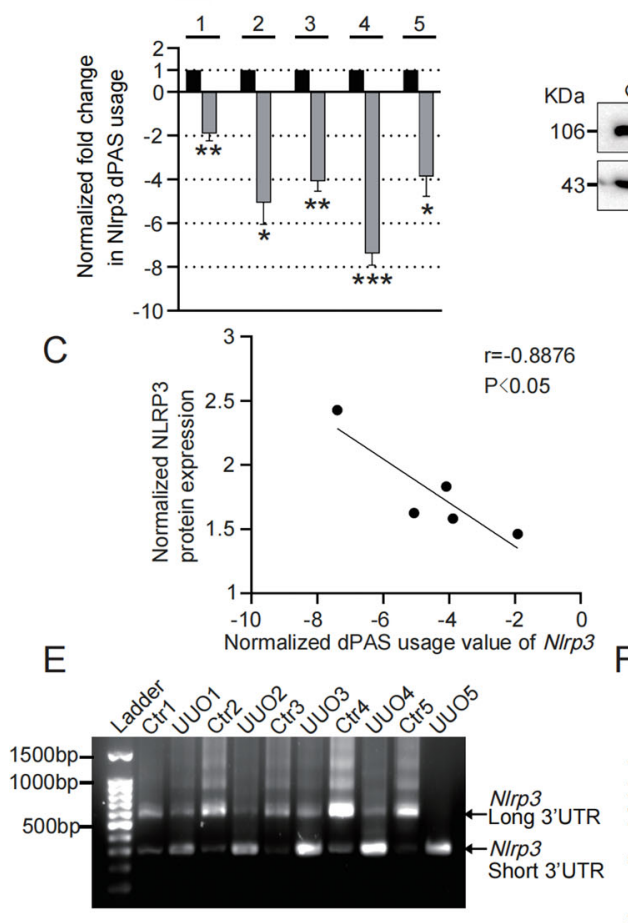

B

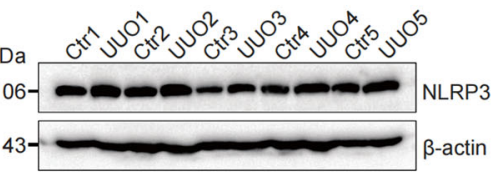

-actin

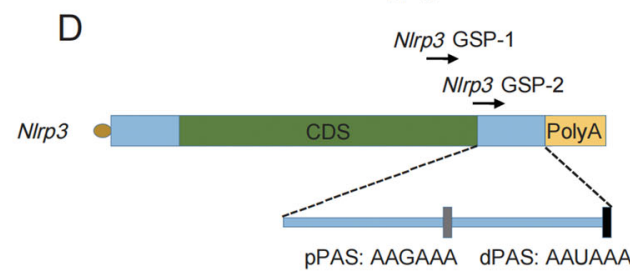

$\square \cup \cup O$
Contralat

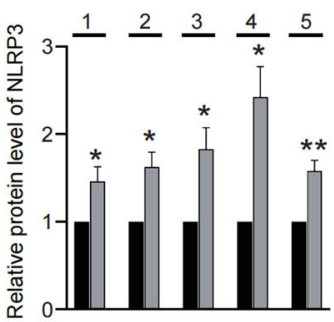

G
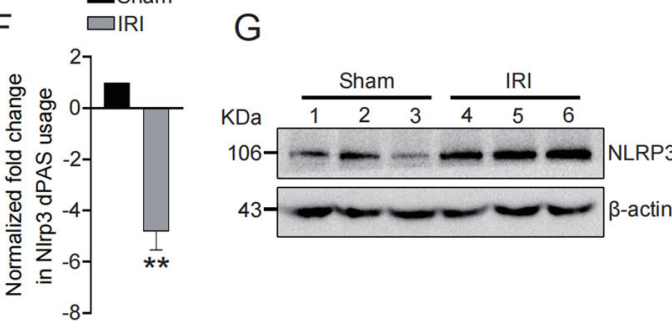

$\mathrm{K}$ $\square \mathrm{H} / \mathrm{R}$
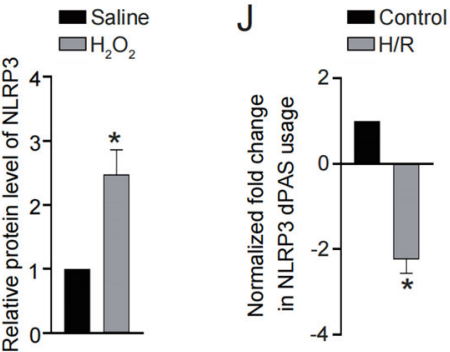

M

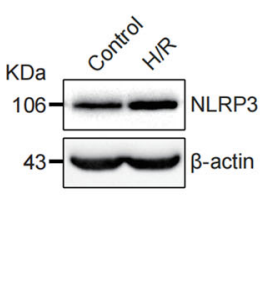

$N L R P 3$ GSP-1
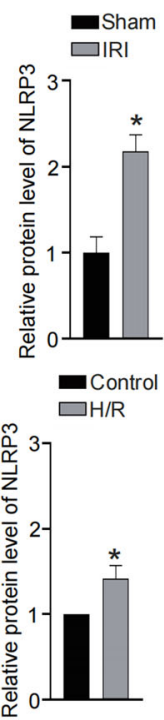
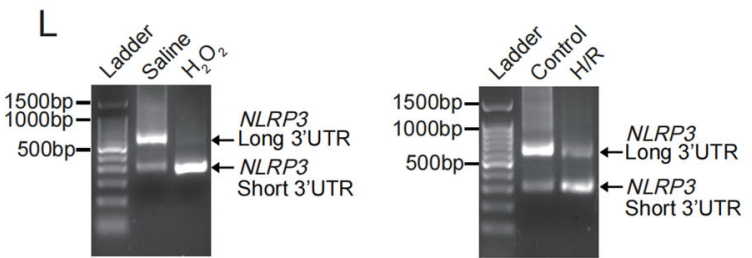
NLRP3 GSP-2

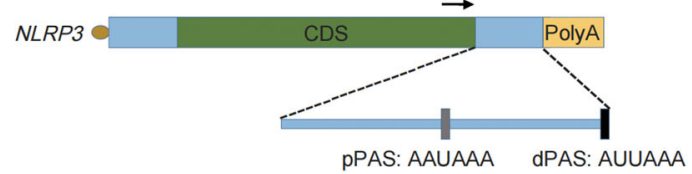

Fig. $13^{\prime}$ UTR of NLRP3 is shortened in response to kidney injury. A qRT-PCR was performed to determine the normalized fold changes of distal PAS usage of NLRP3 in UUO kidneys $(n=5)$ compared to normal kidneys $(n=5)$. B Western blot was performed and quantitatively analyzed to determine the protein levels of NLRP3 in UUO kidneys $(n=5)$ compared to normal kidneys $(n=5)$. C Correlation of normalized dPAS usage value of NLRP3 with normalized protein levels of NLRP3. D The schematic graph indicating the novel PPAS in mice and the primer sequences used in $3^{\prime} R A C E$ assay. Green box, CDS; blue bar, UTR; gray box, the newly-identified PPAS; black box, canonical dPAS. E 3'RACE was performed to determine the expression of mRNA isoforms of NLRP3 in UUO kidneys $(n=5)$ compared to normal kidneys $(n=5)$. $\mathbf{F}$ qRT-PCR was performed to determine the normalized fold changes of distal PAS usage of NLRP3 in IRI kidneys $(n=3)$ compared to normal kidneys $(n=3)$. G Western blot was performed and quantitatively analyzed to determine the protein levels of NLRP3 in IRI kidneys $(n=3)$ compared to normal kidneys $(n=3)$. $\mathbf{H}$ qRT-PCR was performed to determine the normalized fold changes of distal PAS usage of NLRP3 in HK2 cells treated with $\mathrm{H}_{2} \mathrm{O}_{2}$. I Western blot was performed and quantitatively analyzed to determine the protein levels of NLRP3 in HK2 cells treated with $\mathrm{H}_{2} \mathrm{O}_{2}$. $\mathbf{J}$ qRT-PCR was performed to determine the normalized fold changes of distal PAS usage of NLRP3 in HK2 cells treated with H/R. $\mathbf{K}$ Western blot was performed and quantitatively analyzed to determine the protein levels of NLRP3 in HK2 cells treated with H/R. L $3^{\prime}$ RACE was performed to determine the expression of mRNA isoforms of NLRP3 in HK2 cells treated with $\mathrm{H}_{2} \mathrm{O}_{2}$ or H/R. M The schematic graph indicating the novel pPAS in human and the primer sequences used in $3^{\prime}$ RACE assay. Green box, CDS; blue bar, UTR; gray box, the newly-identified pPAS; black box, canonical dPAS. ${ }^{*} P<0.05 ;{ }^{*} P<0.01 ;{ }^{* * *} P<0.001$; All data represent the mean \pm SEM obtained from three independent experiments. Student's $t$ test. 


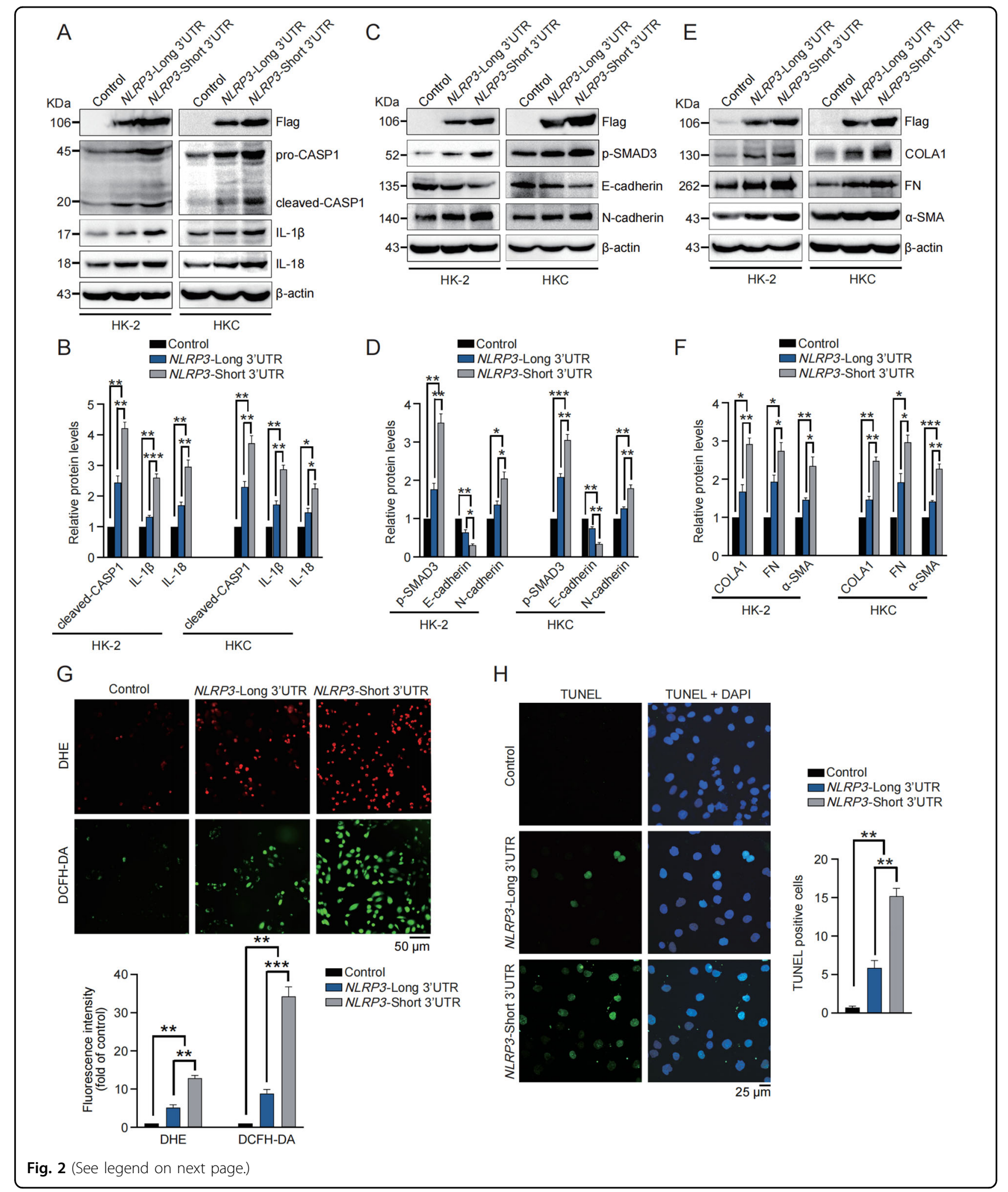

upregulated in all obstructed kidneys to different extents, suggesting NLRP3 $3^{\prime} \mathrm{UTR}$ shortening as a pathological event in kidney injury (Fig. 1A). Consistent with previous studies, protein levels of NLRP3 were upregulated following UUO (Fig. 1B). Particularly, the degrees of NLRP3 $3^{\prime}$ UTR shortening were positively associated with the 
(see figure on previous page)

Fig. 2 3'UTR shortening of NLRP3 amplifies inflammation, fibrogenesis, ROS production, and apoptosis in renal tubular epithelial cells. A Western blot was performed to determine the protein levels of Flag, caspase1, IL-1 $\beta$, and IL-18 in HK2 and HKC cells transfected with empty vector, short 3'UTR NLRP3 or long 3'UTR NLRP3 respectively. B Quantitative analysis of protein levels of caspase1, IL-1 $\beta$ and IL-18 in HK2 and HKC cells transfected with empty vector, short 3'UTR NLRP3 or long 3'UTR NLRP3. C Western blot was performed to determine the protein levels of Flag, phospho-SMAD3, E-cadherin, and N-cadherin in HK2 and HKC cells transfected with empty vector, short 3'UTR NLRP3 or long 3'UTR NLRP3 respectively. D Quantitative analysis of protein levels of phospho-SMAD3, E-cadherin and N-cadherin in HK2 and HKC cells transfected with empty vector, short 3'UTR NLRP3 or long 3'UTR NLRP3. E Western blot was performed to determine the protein levels of Flag, collagen I, fibronectin, and aSMA in HK2 and HKC cells transfected with empty vector, short $3^{\prime} U T R$ NLRP3 or long $3^{\prime} U T R$ NLRP3 respectively. F Quantitative analysis of protein levels of collagen I, fibronectin, and a-SMA in HK2 and HKC cells transfected with empty vector, short 3'UTR NLRP3 or long 3'UTR NLRP3. G Representative images and quantitative analysis of DHE and DCFH-DA staining in HK2 cells transfected with empty vector, short $3^{\prime} U T R$ NLRP3 or long $3^{\prime} U T R$ NLRP3. H Representative images and quantitative analysis of TUNEL staining in HK2 cells transfected with empty vector, short $3^{\prime} U T R$ NLRP3 or long $3^{\prime} U$ TTR NLRP3. ${ }^{*} P<0.05 ;{ }^{* *} P<0.01$; ${ }^{* * *} P<0.001$; All data represent the mean \pm SEM obtained from three independent experiments. Student's $t$ test.

degrees of NLRP3 upregulation (Fig. 1C), suggesting $3^{\prime}$ UTR shortening as important contributor to NLRP3 upregulation in obstructed kidneys. Next, $3^{\prime}$ rapid amplification of cDNA ends ( $\left.3^{\prime} \mathrm{RACE}\right)$ was performed with primers described in Fig. 1D to determine whether NLRP3 3'UTR shortening following UUO derived from usage of novel proximal PAS (pPAS). In all obstructed kidneys, a short $3^{\prime} \mathrm{UTR}$ isoform of NLRP3 was stably found (Fig. 1E). Therefore, a novel proximal PAS (pPAS) corresponding to the short $3^{\prime} \mathrm{UTR}$ isoform of NLRP3 was identified in mice (Fig. 1D). Similarly, in IRI kidneys, obvious $3^{\prime}$ UTR shortening of NLRP3 was also observed, as well as enhanced protein levels of NLRP3 (Fig. 1F, G).

In order to confirm whether $3^{\prime}$ UTR shortening of NLRP3 existed in human, the human renal proximal tubular epithelial cells HK2 were treated with $\mathrm{H}_{2} \mathrm{O}_{2}$ or hypoxia/reoxygenation $(\mathrm{H} / \mathrm{R})$ to simulate UUO $\left(\right.$ ref. $^{25}$ ) or IRI (ref. ${ }^{1}$ ) model in vitro. Both NLRP3 $3^{\prime}$ UTR shortening and NLRP3 upregulation were induced by stimulation of $\mathrm{H}_{2} \mathrm{O}_{2}$ or $\mathrm{H} / \mathrm{R}$ (Fig. $1 \mathrm{H}-\mathrm{K}$ ). Furthermore, 3'RACE was performed and showed that in HK2 cells treated with $\mathrm{H}_{2} \mathrm{O}_{2}$ or $\mathrm{H} / \mathrm{R}$, an unreported short $3^{\prime} \mathrm{UTR}$ isoform of NLRP3 appeared (Fig. 1L), identifying a novel pPAS in human (Fig. 1M). These results demonstrated NLRP3 $3^{\prime}$ UTR is shortened in response to kidney injury, and such 3'UTR shortening associates with NLRP3 upregulation, suggesting a key role of APA regulation in NLRP3 overactivation and kidney injury progression.

\section{3'UTR shortening of NLRP3 amplifies inflammation, fibrogenesis, ROS production and apoptosis in renal tubular epithelial cells}

We thus continued to investigate the function of NLRP3 $3^{\prime}$ UTR shortening in vitro. Considering the canonical function of NLRP3 (ref. ${ }^{26}$ ), the effects of NLRP3 $3^{\prime}$ UTR shortening in inflammasome activation was examined. HK2 and HKC cells were transfected with newly-identified short-3'UTR isoform of NLRP3 or long-3'UTR isoform of NLRP3 (namely full-length $N L R P 3)$ respectively, and significantly enhanced expression of caspase1, IL-1 $\beta$ and IL-18 was observed in both cells transfected with short-3'UTR NLRP3, compared with those transfected with full-length NLRP3 or empty vector (Fig. 2A, B), indicating the amplified inflammasome activation induced by NLRP3 3'UTR shortening.

Independently of inflammasome, NLRP3 also functions in fibrogenesis to aggravate kidney injury via activating TGF- $\beta$ pathway ${ }^{27}$. As evidenced by the stronger stimulation of phosphorylated SMAD3 (Fig. 2C, D), TGF- $\beta$ pathway was found more activated upon transfection of short-3'UTR NLRP3. Accordingly, short-3'UTR NLRP3 displayed advantages in promoting fibrogenesis (Fig. 2E, F). More importantly, NLRP3 was reported to trigger ROS production, and DHE/DCFH-DA staining showed ROS production benefited from NLRP3 $3^{\prime} \mathrm{UTR}$ shortening (Fig. 2G). Finally, we evaluated the effect of NLRP3 $3^{\prime}$ UTR shortening on cell apoptosis and found short-3'UTR NLRP3 caused more cell apoptosis than the full-length one did (Fig. 2H). These in vitro evidences strongly suggested the pathological role of NLRP3 3'UTR shortening in kidney injury.

\section{3'UTR shortening of NLRP3 benefits MRNA stability and leads to enhanced protein expression of NLRP3}

Since the pathological role of NLRP3 3'UTR shortening probably relied on increasing NLRP3 protein level, we thus started to uncover how $3^{\prime}$ UTR shortening dictated the final expression of NLRP3. Luciferase activity assay demonstrated the reporter plasmid with short $3^{\prime}$ UTR of $N L R P 3$ gained an augmented luciferase activity in both cells (Fig. 3A). Furthermore, the mRNA decay time course was performed with a $3^{\prime} \mathrm{UTR}$ isoform-specific qRT-PCR strategy $^{28}$. A distinct diversity of half-life between short and long 3'UTR of NLRP3 was observed (Fig. 3B, C), indicating the superiority of short-3'UTR NLRP3 in mRNA stability.

MicroRNA-mediated silencing plays a decisive role in mRNA stability, so we hypothesized $3^{\prime}$ UTR shortening changed the response of NLRP3 mRNA to microRNAs. miR-223-3p and miR-22 have been frequently reported to target NLRP3 in various settings ${ }^{18,19}$, and in particular, 
A

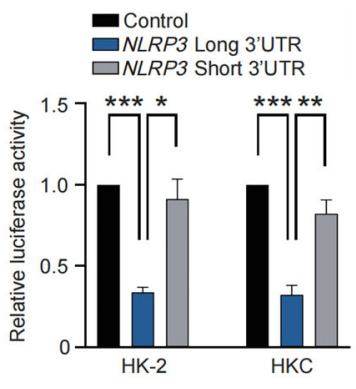

C

-NLRP3-Long 3'UTR $\square N L R P 3$-Short 3'UTR

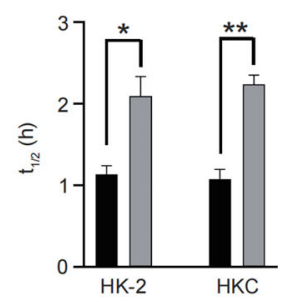

E

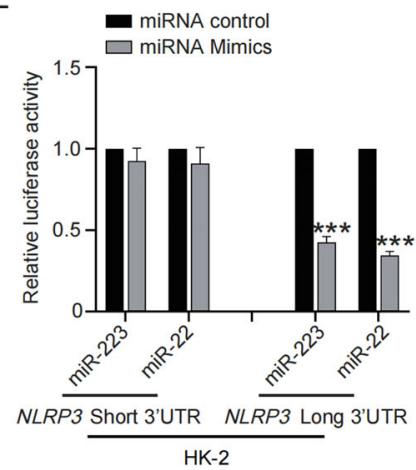

B

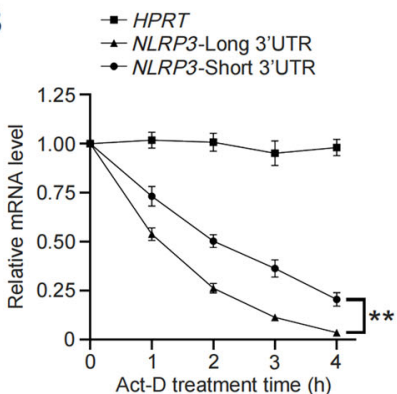

HK-2

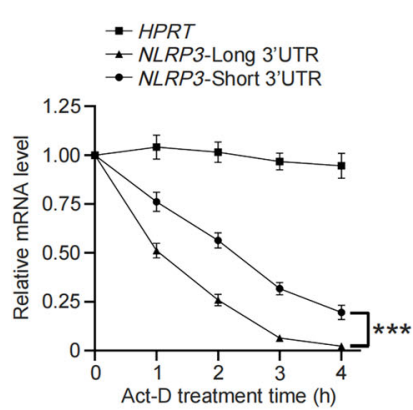

HKC

D

NLRP3 3'UTR mutant-1 5'...GCUAUCUUUCUAUUAUGUCUGCA...3'

miRNA-223-3p 3' ACCCCAUAAACUGUUUGACUGU 5'

NLRP3 3'UTR 5'...GCUAUCUUUCUAUUAACUGACCA...3'

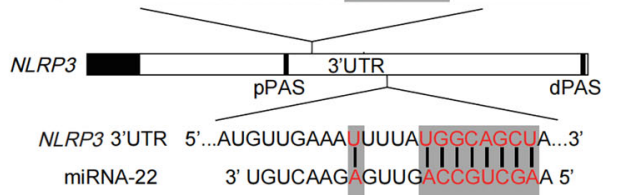

NLRP3 3'UTR mutant-2 5'...AUGUUGAAAUUUUAUCCGACGAA...3'

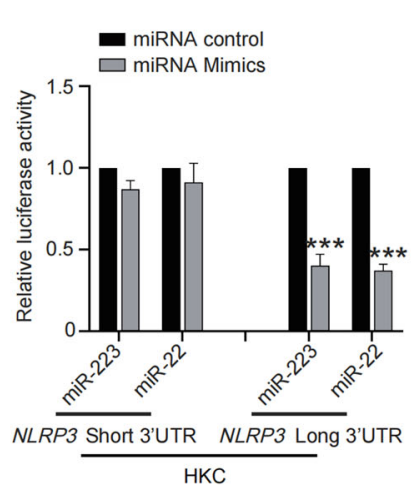

$\mathrm{F}$ NLRP3 Long WT 3'UTR
NLRP3 3'UTR mutant-1
NLRP3 3'UTR mutant-2

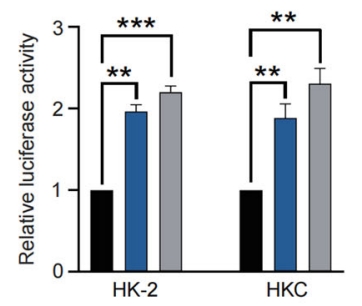

Fig. 3 3'UTR shortening of NLRP3 benefits mRNA stability and leads to enhanced protein expression of NLRP3. A Luciferase reporter assay was performed in HK2 and HKC cells to examine luciferase activity produced by reporters carrying short $3^{\prime} U T R$ or long $3^{\prime} U T R$ of NLRP3 respectively. B Isoform-specific qRT-PCR was performed to determine long-3'UTR and short-3'UTR NLRP3 mRNA half-life in HK2 and HKC cells treated with Act-D. Total RNA was normalized with HPRT. C The bar graph showing the half-life in hours calculated for long-3'UTR NLRP3 and short-3'UTR NLRP3. D Schematic diagram showing the binding sites of miRNAs in NLRP3 $3^{\prime} U$ TR and the mutant NLRP3 used in F. E Luciferase reporter assay was performed to examine luciferase activity produced by reporters carrying short 3'UTR or long 3'UTR of NLRP3 respectively in HK2 and HKC cells cotransfected with mimics of miR-223 or miR-22. $\mathbf{F}$ Luciferase reporter assay was performed to examine luciferase activity produced by reporters carrying long $3^{\prime} U T R$ of NLRP3 or mutants of long $3^{\prime} U$ UTR of NLRP3 respectively. ${ }^{*} P<0.05$; ${ }^{* *} P<0.01$; ${ }^{* *} P<0.001$; All data represent the mean \pm SEM obtained from three independent experiments. Student's $t$ test.

both of their binding sites on NLRP3 $3^{\prime} \mathrm{UTR}$ were downstream of the newly-identified pPAS (Fig. 3D). Luciferase reporter plasmids with short $3^{\prime} \mathrm{UTR}$ or long $3^{\prime} \mathrm{UTR}$ of NLRP3 were co-transfected with mimics of miR-223-3p or miR-22. In both cells, the miR-223-3/ miR-22 mimics weakened luciferase activity of long $N L R P 33^{\prime}$ UTR, in contrast, they showed no significant effects on luciferase activity of short NLRP3 $3^{\prime} \mathrm{UTR}$ (Fig. 3E). On the other hand, the luciferase plasmid carrying mutated long 3'UTR of NLRP3 (Fig. 3D) displayed an enhanced luciferase activity, compared with the plasmid carrying the wild-type (Fig. 3F). Therefore, these data indicated depending on $3^{\prime} \mathrm{UTR}$ shortening, NLRP3 mRNA is stabilized via evading miRNA-mediated silencing, leading to NLRP3 upregulation.

\section{FIP1 binds to NLRP3 3'UTR via C-terminal arginine-rich domain and induces $3^{\prime}$ UTR shortening of NLRP3}

The events of $3^{\prime} \mathrm{UTR}$ alteration in most cases are the results of aberrant expression of APA trans-factors ${ }^{23}$, including CSTF2, CPSF1, FIP1, CFIm59, CFIm25 and PABPN1. Therefore, in order to determine the factor 
governing NLRP3 $3^{\prime} \mathrm{UTR}$ shortening, the correlations between the 3'UTR length of NLRP3 and transfection of the above-mentioned APA trans-factors were examined. In HK2 cells FIP1 was found to predominantly induce NLRP3 3'UTR shortening (Fig. 4A). Moreover, only CSTF2 (ref. ${ }^{23}$ ), FIP1 (ref. ${ }^{29}$ ) and CFIm59 (ref. ${ }^{30}$ ) have been reported to induce $3^{\prime} \mathrm{UTR}$ shortening, then $3^{\prime} \mathrm{RACE}$ was performed and showed among three candidates, FIP1 gained the strongest capability in upregulating short $3^{\prime}$ UTR isoform of NLRP3 (Fig. 4B), confirming FIP1 as upstream governor of NLRP3 $3^{\prime}$ UTR shortening. Furthermore, western blot and immunofluorescence (IF) staining showed FIP1 upregulated NLRP3 expression in HK2 cells (Fig. 4C, D).

In order to confirm FIP1 as the bona fide inducer of NLRP3 3'UTR shortening, RNA immunoprecipitation (RIP) was used to determine which region of NLRP3 $3^{\prime}$ UTR FIP1 binds to. As demonstrated by qRT-PCR, FIP1 was found to predominantly associate with the region around NLRP3 pPAS, rather than that around dPAS (Fig. 4E). We continued to map the functional domain of FIP1 mediating the binding between FIP1 and NLRP3 mRNA. The C-terminal arginine-rich domain (Fig. 4F) was previously reported to mediate the binding of FIP1 and $\mathrm{mRNA}^{31}$, therefore two recombined truncated FlagFIP1 (1-488 aa and 489-594 aa) were purified and used for RIP. The RIP assay confirmed FIP1 depended on its arginine-rich (R) domain to bind with NLRP3 mRNA (Fig. 4G). The above results clearly demonstrated FIP1 as the inducer of NLRP3 $3^{\prime}$ UTR shortening.

FIP1 is upregulated in injured kidneys and CKD specimens

We thus studied the role of FIP in kidney injury. To start with, the expression of FIP1 in the five pairs of UUO kidneys and matched unobstructed kidneys which were previously used, was examined. As shown by western blot, FIP1 was overexpressed in UUO kidneys (Fig. 4H). Moreover, the expression of FIP1 was positively associated with NLRP3 $3^{\prime} \mathrm{UTR}$ shortening and NLRP3 expression (Fig. 4I, J). Additionally, IF imaging again confirmed the overexpression of FIP1 in both UUO-induced and IRIinduced injured kidneys (Fig. 4K).

Since aggravated kidney injury is very likely to turn into CKD, we investigated the expression of FIP1 in clinical CKD specimens to determine whether FIP1 is a contributor of AKI-CKD transition. The expression of FIP1 in 27 kidney specimens from CKD patients was examined by immunohistochemistry (IHC) staining (Fig. 4L), and these specimens were divided into a group of high FIP1 expression $(n=17)$ and another group of low FIP1 expression $(n=10)$. Importantly, high FIP1 expression negatively associated with eGFR $(P<0.05 ;$ Fig. $4 \mathrm{M})$. Besides, the patients with high FIP1 expression seemed to have a relatively higher percentage of proteinuria than those with low FIP1 expression did (Fig. 4N). Taken together, these results suggested FIP1 as a harmful role in kidney injury and contributor of AKI-CKD transition.

\section{FIP1 promotes inflammation, fibrogenesis, ROS production} and apoptosis in renal tubular epithelial cells

Next, the function of FIP1 in renal tubular epithelial cells was investigated. First, transfection of Flag-FIP1 strongly upregulated the expression of caspase1, IL-1 $\beta$ and IL-18, indicating FIP1's capability of activating inflammasome (Fig. 5A, B). Furthermore, TGF- $\beta$ pathway and fibrogenesis were stimulated by enforced expression of FIP1 in both cells (Fig. 5C-F). Besides, ROS production was enhanced in HK2 cells by Flag-FIP1 transfection, as shown by DHE and DCFH-DA staining (Fig. 5G). Finally, TUNEL staining confirmed the pro-apoptotic role of FIP1 in HK2 cells (Fig. 5H). Therefore, these results showed that FIP1 behaves as a pro-injury role in kidney tubular cells, suggesting FIP1 as a promising therapeutic target for kidney injury.

\section{FIP1 is upregulated by oxidative stress and required for oxidative stress-induced NLRP3 upregulation, inflammation, apoptosis and cell damage}

Since we found $3^{\prime}$ UTR shortening of NLRP3 was induced by oxidative stress and governed by APA trans-factor FIP1, we wondered whether oxidative stress functioned upstream of FIP1. We thus treated HK2 cells with $\mathrm{H}_{2} \mathrm{O}_{2}$ or $\mathrm{H} / \mathrm{R}$ and found FIP1 was obviously upregulated by both treatments (Fig. 6A, B), suggesting oxidative stress may depend on upregulating FIP1 to induce NLRP3 3'UTR shortening. To prove this, FIP1 was silenced in HK2 cells, followed by treatment of $\mathrm{H}_{2} \mathrm{O}_{2}$ or $\mathrm{H} / \mathrm{R}$. Indeed, FIP1 silencing reversed the $3^{\prime} \mathrm{UTR}$ shortening of NLRP3 which was induced by treatment of $\mathrm{H}_{2} \mathrm{O}_{2}$ or $\mathrm{H} / \mathrm{R}$ (Fig. 6C, D).

In kidney injury, oxidative stress mainly functions through promoting inflammation, apoptosis ${ }^{6,32}$. In particular, oxidative stress acts as a key stimulator of NLRP3 inflammasome activation ${ }^{33}$. We thus hypothesized FIP1 might mediate the oxidative stress-induced NLRP3 inflammasome activation. Significantly, FIP1 silencing abolished NLRP3 upregulation and subsequent inflammasome activation induced by $\mathrm{H}_{2} \mathrm{O}_{2}$ or $\mathrm{H} / \mathrm{R}$ (Fig. 6E, F). In addition, $\mathrm{H} / \mathrm{R}$ stimulation-induced tubular cell apoptosis and damage were both mitigated by FIP1 silencing (Fig. 6G, H). These results emphasized the importance of FIP1 and APA regulation in oxidative stress-induced kidney injury.

\section{Knockdown (KD) of FIP1 alleviates UUO-induced fibrogenesis, inflammation activation, oxidative stress and apoptosis}

To probe into the therapeutic potential of FIP1 in kidney injury in vivo, FIP1 was knocked down via adeno- 


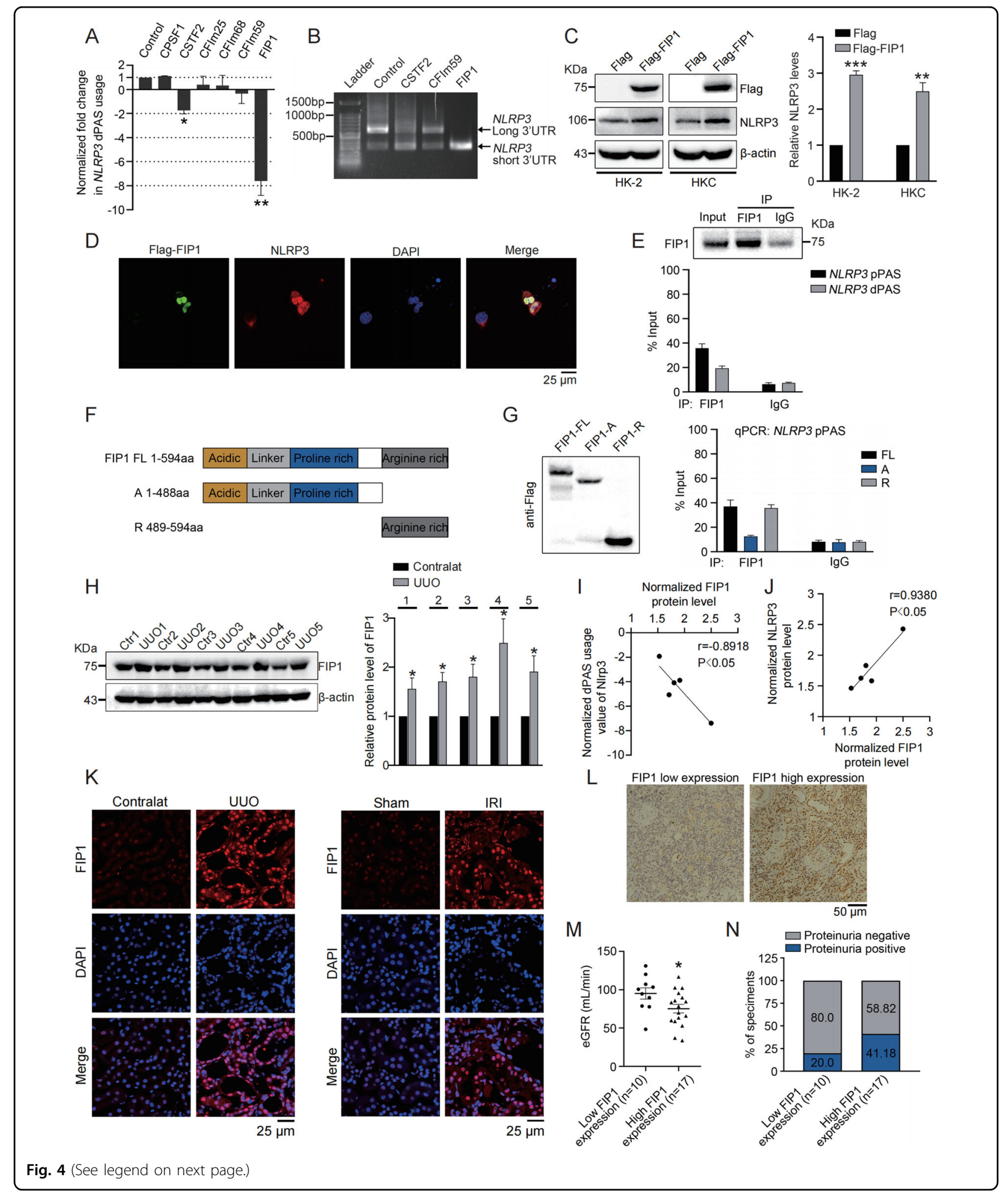

associated virus-9 (AAV9) packed shRNA-FIP1, followed by construction of 14-day UUO (Fig. 7A). As demonstrated by Masson's trichrome staining,
Picrosirius Red staining and IHC staining, the UUOinduced kidney fibrosis was clearly alleviated by FIP1 KD (Fig. 7B, C). Additionally, the IHC staining for NLRP3 
(see figure on previous page)

Fig. 4 FIP1 binds to NLRP3 mRNA via C-terminal arginine-rich domain to induce $3^{\prime}$ UTR shortening of NLRP3 and is upregulated in injured kidneys and CKD specimens. A qRT-PCR was performed to determine the normalized fold changes of distal PAS usage of NLRP3 in HK2 cells transfected with each indicated APA trans-factor. B 3'RACE was performed to determine the expression of mRNA isoforms of NLRP3 in HK2 cells transfected with CSTF2, CFIm59, or FIP1 respectively. C Western blots were performed and quantitatively analyzed to detect protein levels of NLRP3 in HK2 and HKC cells transfected with Flag-FIP1 or empty vector. D Immunofluorescence staining was performed to detect protein levels of NLRP3 in HK2 cells transfected with Flag-FIP1. E RNA immonuprecipitation (RIP) assay was performed to determine the region of NLRP3 3'UTR which FIP1 binds to. And GRT-PCR was performed to quantify the relative enrichment of NLRP3 3'UTR regions associated with FIP1 relative to the input. IgG was used as a control. F Schematic diagram showing the truncated regions of FIP1 used for RIP assay. G RIP assay was performed to map the binding region of FIP1 to NLRP3 $3^{\prime} U T R$. And qRT-PCR was performed to quantify the relative enrichment of NLRP3 mRNA levels associated with each truncated FIP1 relative to the input. IgG was used as a control. $\mathbf{H}$ Western blot was performed and quantitatively analyzed to determine the protein levels of FIP1 in UUO kidneys $(n=5)$ compared to normal kidneys $(n=5)$. I Correlation of normalized protein levels of FIP1 with normalized dPAS usage value of NLRP3. J Correlation of normalized protein levels of FIP1 with normalized protein levels of NLRP3. K Immunofluorescence staining was performed to detect protein levels of FIP1 in UUO or IRI kidneys compared to normal kidneys. L Representative images of IHC staining for FIP1 in FIP1 high-expressed and FIP1 low-expressed CKD specimens. M The scatter plot demonstrating the eGFR of patients with high FIP1 expression $(n=17)$ and those with low FIP1 expression $(n=10)$. N The correlation analysis between FIP1 expression and proteinuria in CKD patients. ${ }^{* *} P<0.01$; ${ }^{* *} P<0.001$; All data represent the mean \pm SEM obtained from three independent experiments. Student's $t$ test.

offered the in vivo evidence for FIP1-induced NLRP3 upregulation (Fig. 7D, E). Moreover, the infiltrating of T cells and macrophages was mitigated in FIP1 KD mice (Fig. 7D, E), reflecting the role of FIP1 in inflammation activation in vivo. Finally, the augmented levels of ROS and apoptosis in UUO kidneys were relieved by FIP1 KD (Fig. 7F, G).

\section{FIP1 KD alleviates IRI-induced inflammation activation, oxidative stress and apoptosis}

We next examined the therapeutic potential of FIP1 in acute kidney injury in vivo, and the 2-day IRI was performed following FIP1 KD (Fig. 8A). IRI even induced a more significant upregulation of NLRP3 than UUO did, but FIP1 KD strongly reversed such NLRP3 upregulation (Fig. 8B, C). In particular, IHC staining for CD11b and F4/80 showed that FIP1 was instrumental in inflammation activation in IRI kidneys (Fig. 8B, C). Furthermore, less IRI-induced apoptosis was witnessed in FIP1 KD kidneys (Fig. 8D). Finally, DHE staining and measurement of MDA/SOD indicated the oxidative stress following IRI was reduced by FIP1 KD (Fig. 8E, F). Taken together, targeting FIP1 is a promising therapy for kidney injury.

\section{Discussion}

Kidney injury is very much likely to transit into CKD and even kidney failure. Moderate inflammation is renoprotective role limiting the kidney damage. In contrast, amplified inflammation converts into an initiator of various pathological effects, especially apoptosis, oxidative stress, and fibrogenesis. Therefore, to inhibit the amplification of inflammation and subsequent pathological effects is an ideal strategy for kidney injury.

In most cases, NLRP3 upregulation is responsible for amplified inflammation and other pathological effects. To date, the upregulation of NLRP3 has been attributed to its enhancement of transcription or protein stability ${ }^{34,35}$. In this study, we reported for the first time that, APA-induced enhancement of NLRP3 mRNA stability, is also a vital mechanism for NLRP3 upregulation. We found $3^{\prime} U T R$ shortening stimulated NLRP3 upregulation via enhancing NLRP3 mRNA stability. Such $3^{\prime}$ UTR shortening-induced NLRP3 upregulation caused amplified inflammation, as well as enhanced apoptosis, ROS production and fibrogenesis, leading to kidney injury progression and AKI-CKD transition (Fig. 8G). These findings indicated $3^{\prime} \mathrm{UTR}$ shortening of NLRP3 as a vital event of kidney injury progression, offering a new therapeutic strategy for kidney injury and other inflammation-associated diseases.

Oxidative stress is a well-recognized pathological factor in kidney injury, therefore a better understanding of how it exerts the po-injury effects in kidney will greatly benefit the development of treatment for kidney injury. In this study, the indispensable crosstalk between oxidative stress and APA was uncovered for the first time, especially in kidney injury progression. First, we found oxidative stressinduced upregulation of APA trans-factor FIP1 and further $3^{\prime} \mathrm{UTR}$ shortening of NLRP3, leading to amplification of multiple pathophysiological effects in kidney injury. It is worth noting that oxidative stress upregulated FIP1 expression, and in return FIP1 upregulation enhanced the oxidative stress. FIP1 thus formed a positive regulatory loop with oxidative stress in kidney injury progression. Second, inhibition of NLRP3 $3^{\prime} \mathrm{UTR}$ shortening via silencing FIP1 strongly reversed the inflammation activation and apoptosis induced by oxidative stress. Based on the above evidence, this study built the first bridge between oxidative stress and APA. Besides, previous studies found oxidative stress-induced inflammasome activation via stimulating NLRP3 transcription ${ }^{33}$. Here we uncovered that via strengthening mRNA stability is 


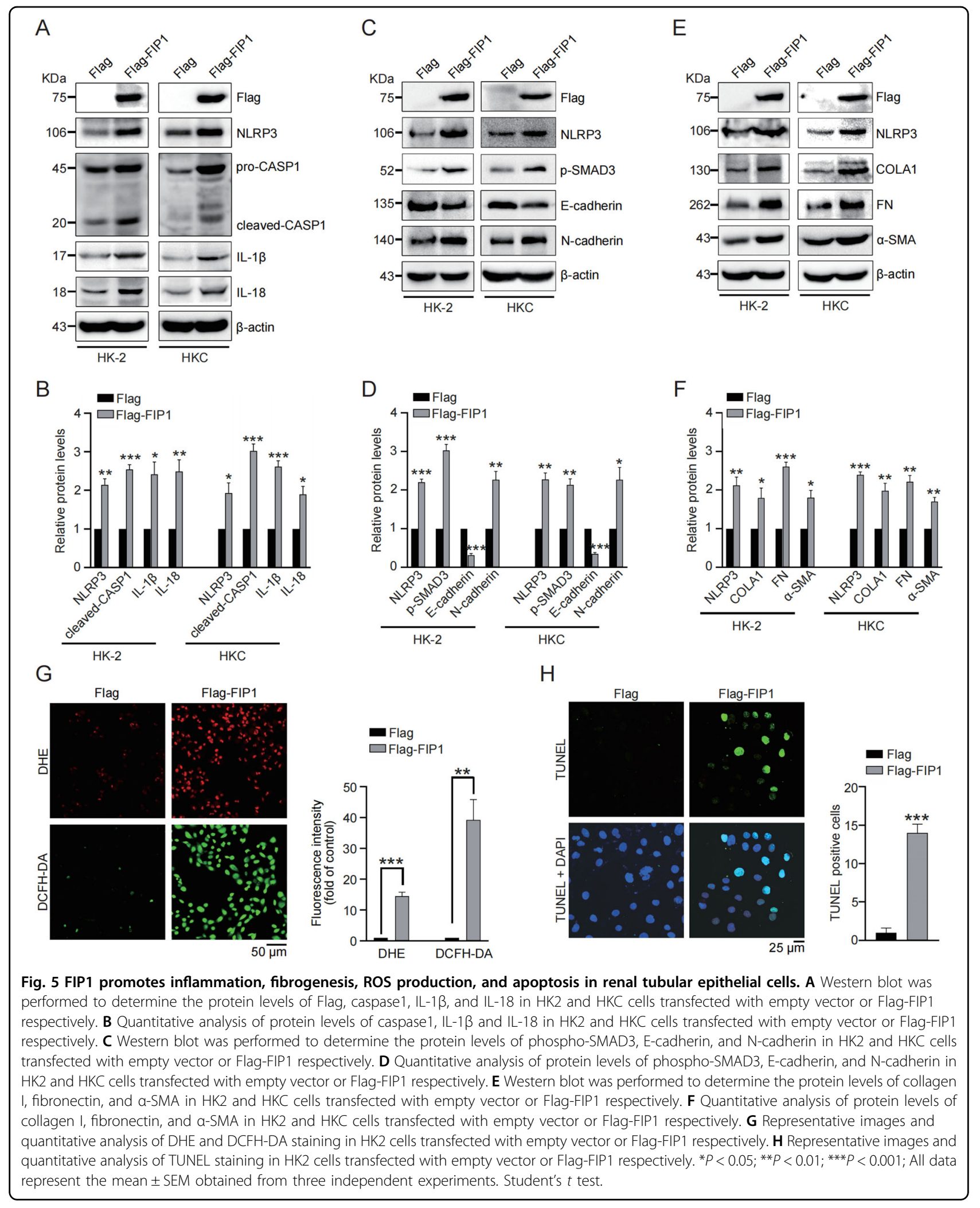




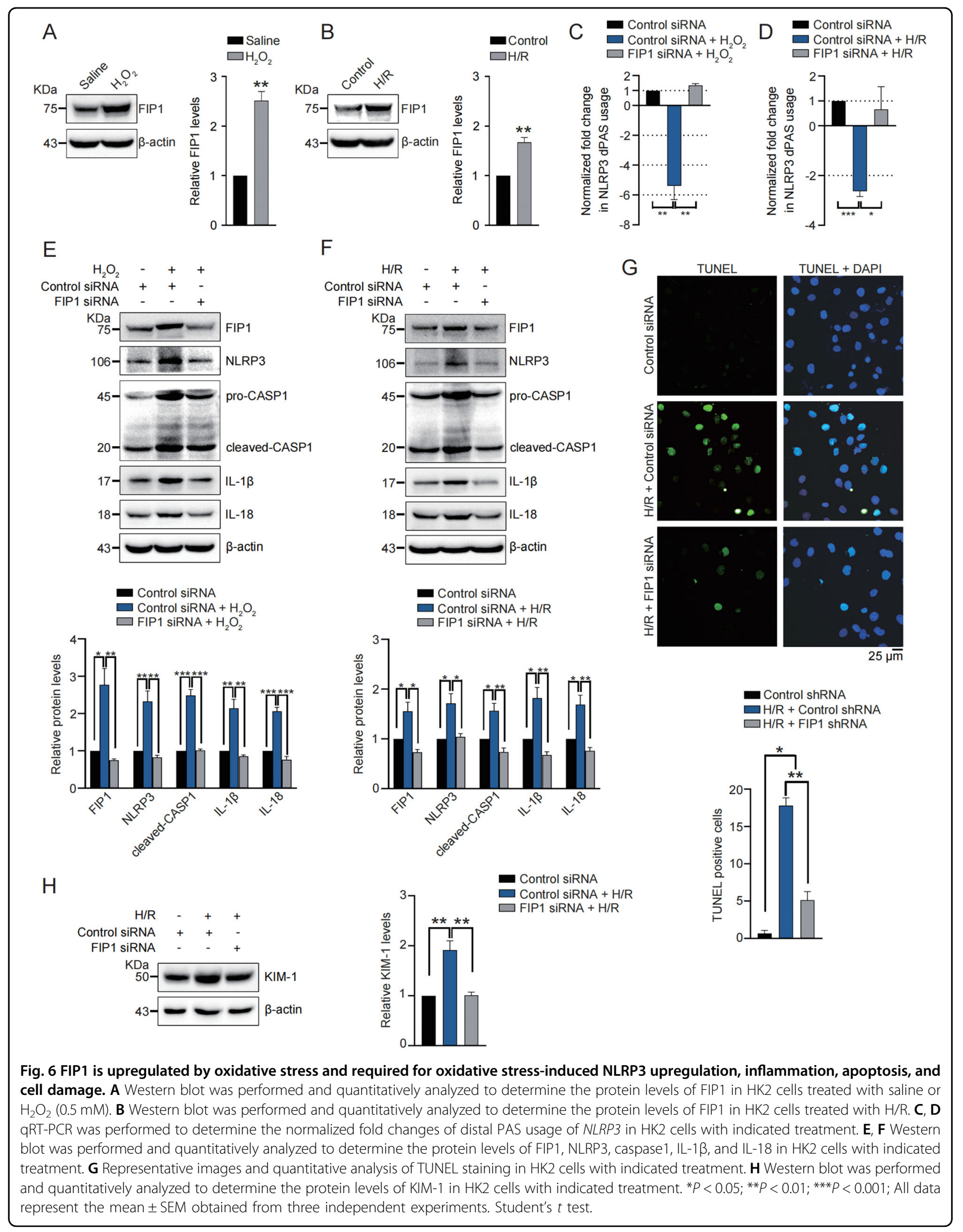




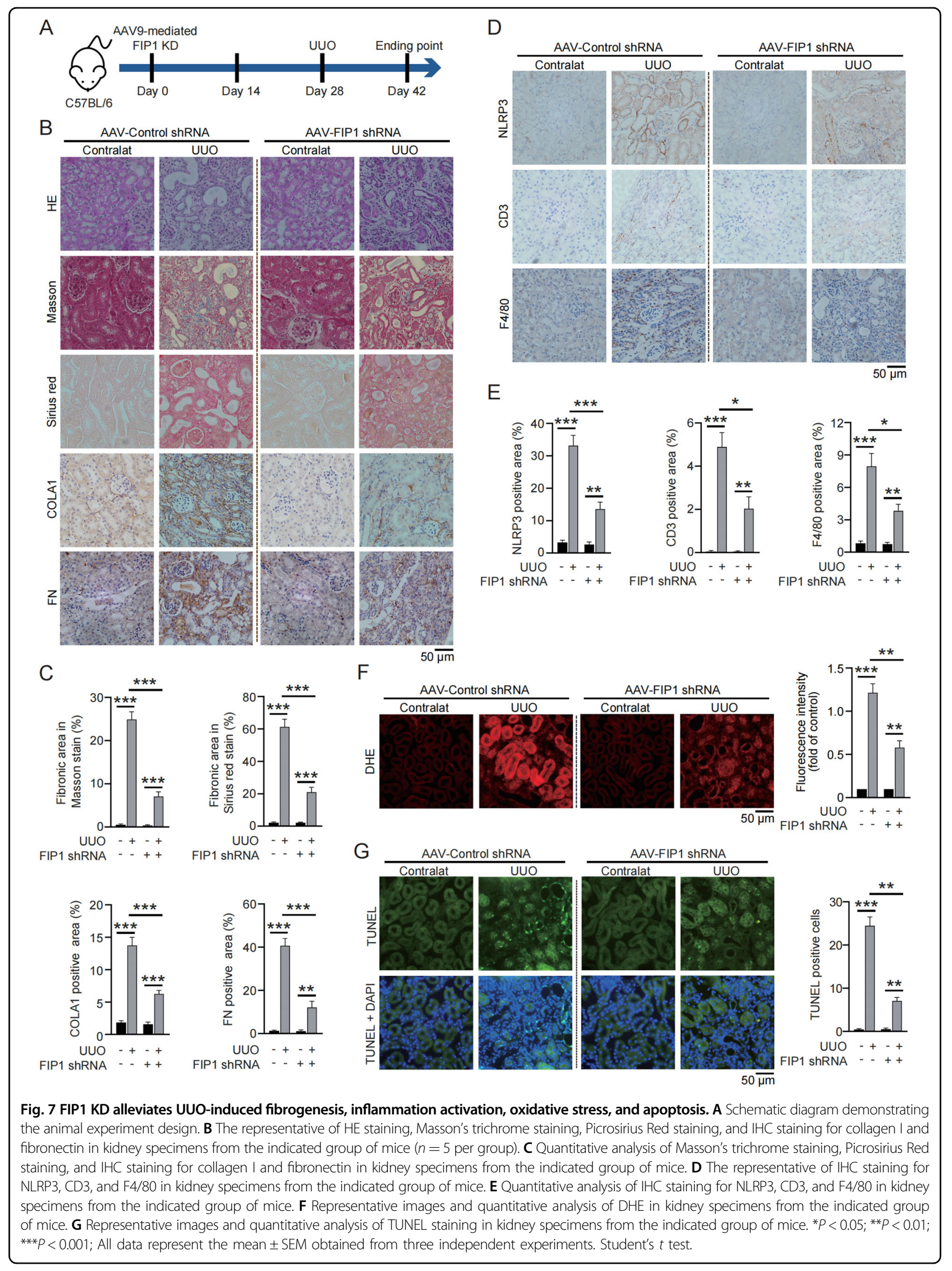



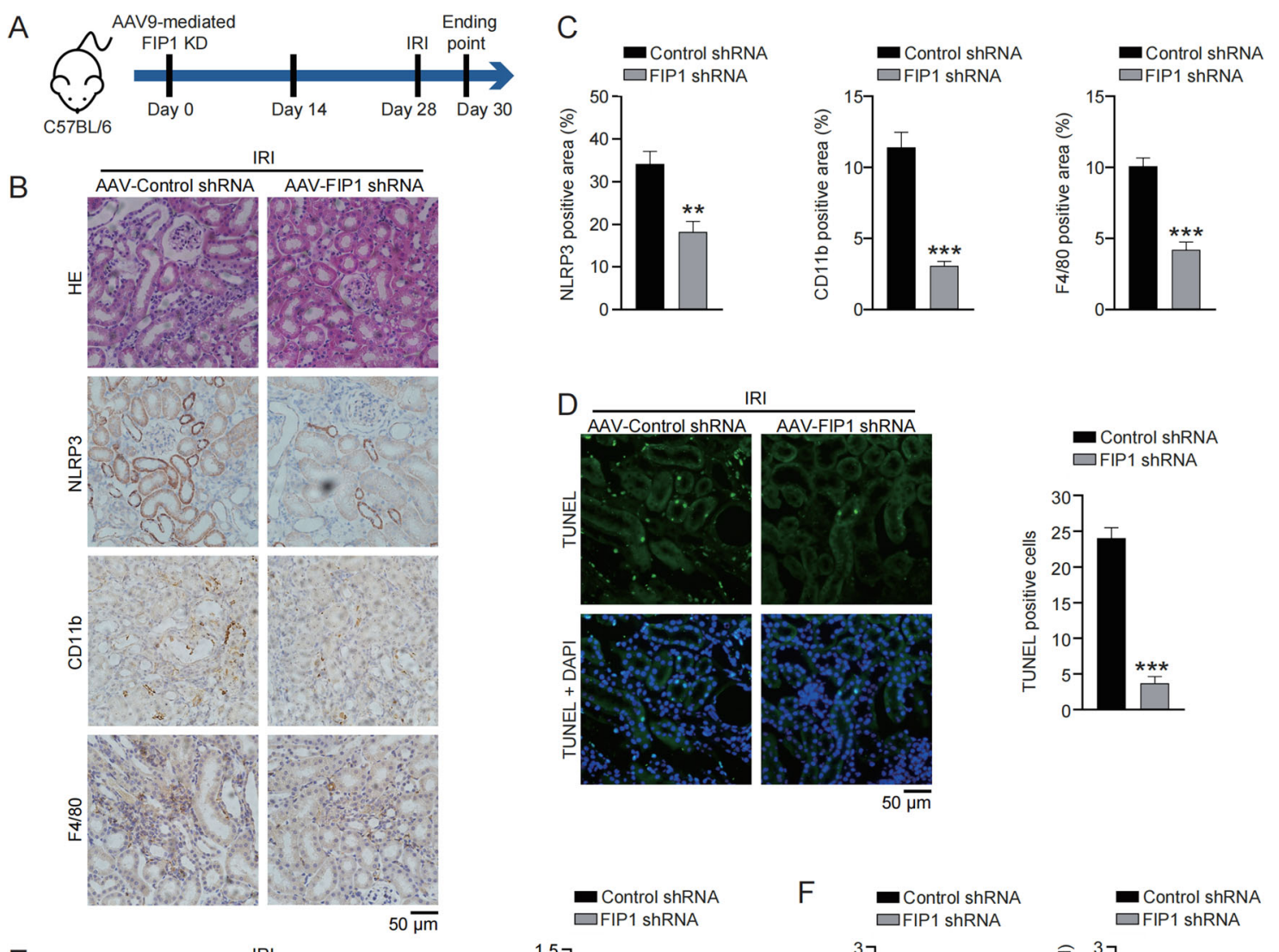

$E$
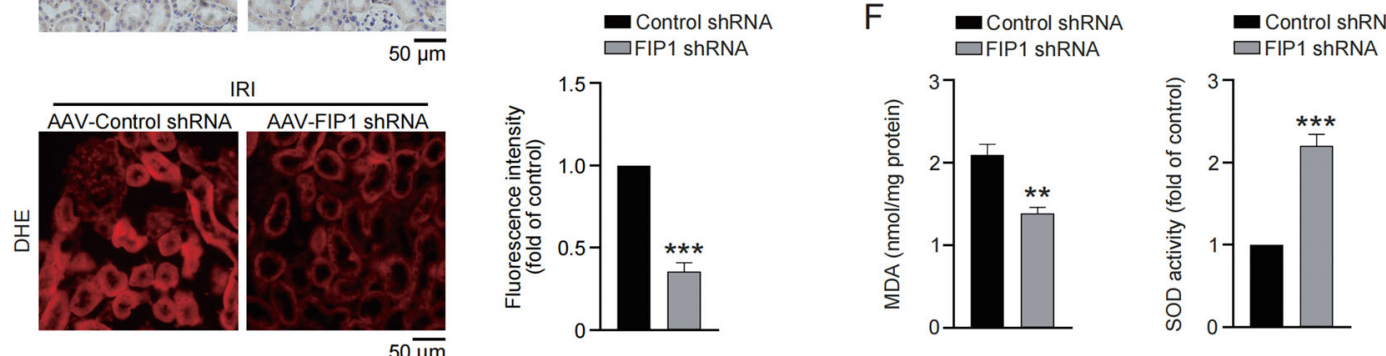

G

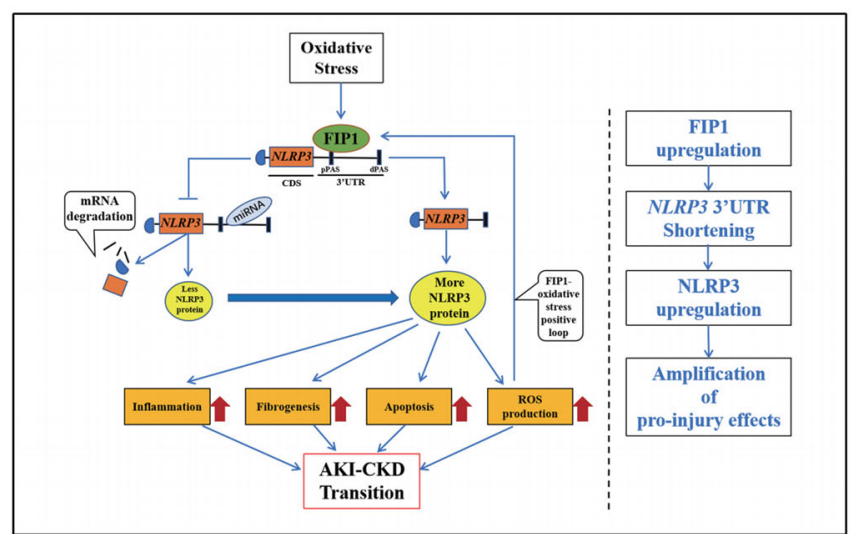

Fig. 8 FIP1 KD alleviates IRI-induced inflammation activation, oxidative stress, and apoptosis. A Schematic diagram demonstrating the animal experiment design. B The representative of HE staining and IHC staining for NLRP3, CD11b, and F4/80 in kidney specimens from the indicated group of mice ( $n=5$ per group). C Quantitative analysis of IHC staining for NLRP3, CD11b, and F4/80 in kidney specimens from the indicated group of mice. D Representative images and quantitative analysis of TUNEL staining in kidney specimens from the indicated group of mice. E Representative images and quantitative analysis of DHE staining in kidney specimens from the indicated group of mice. F Quantitative analysis of MDA (left) and SOD (right) of mice from the indicated group. G Proposed model of FIP1-induced kidney injury progression and AKI-CKD transition. $P<0.05$; ${ }^{* *} P<0.01$; ${ }^{* * *} P<$ 0.001; All data represent the mean \pm SEM obtained from three independent experiments. Student's $t$ test. 
another key mechanism for oxidative stress to promote NLRP3 upregulation and inflammasome activation.

Furthermore, we identified the APA trans-factor FIP1 as the upstream regulator governing $3^{\prime} \mathrm{UTR}$ shortening in kidney injury. We confirmed FIP1 predominantly bound to pPAS of NLRP3 3'UTR depending on its arginine-rich domain, inducing NLRP3 $3{ }^{\prime} \mathrm{UTR}$ shortening and NLRP3 overexpression. Moreover, FIP1 was strongly upregulated in UUO and IRI models. More importantly, FIP1 was overexpressed in clinical specimens of CKD and negatively associated with renal function of CKD patients. In vivo, FIP1 KD indeed alleviated the inflammation, oxidative stress, apoptosis, and fibrogenesis induced by UUO and/or IRI. FIP1 was found to be instrumental in self-renewal of stem cells, and no pathological role of FIP1 was identified. Therefore, this study uncovered its first pathological function. FIP1 may be a determinant of AKI-CKD transition and promising therapeutic target for kidney injury, highlighting the importance of the development of therapies targeting APA regulation, especially FIP1.

FIP1 is a well-known APA trans-factor, as well as the most important component of Cleavage and Polyadenylation Specificity Factor (CPSF) complex. Among the identified APA trans-factors, CFIm59, CSTF2, and FIP1 are the only ones proved to induce $3^{\prime} \mathrm{UTR}$ shortening. CSTF2 and CFIm59 unidirectionally function in favoring proximal PAS and inducing $3^{\prime} \mathrm{UTR}$ shortening. In comparison, of note, FIP1 was reported to favor distal PAS when the alternative PASs were close to one another ${ }^{29}$. However, inconsistent with the abovementioned study, in the case of NLRP3, whose alternative PASs are close to one another, FIP1 still favored proximal PAS and induced $3^{\prime}$ UTR shortening. Therefore, the actual function model of FIP1 in APA needs more intensive investigations.

Aberrant expression of NLRP3 is responsible for overactivated inflammation and subsequent onset of disease $^{35,36}$. However, to date, NLRP3 upregulation is mostly found to be associated with enhancement of transcriptional activity or protein stability of NLRP3. In this study we first reported that enhanced mRNA stability derived from APA regulation, was also an important source of NLRP3 overexpression, suggesting to inhibit the $3^{\prime} \mathrm{UTR}$ shortening of NLRP3 could be a promising therapeutic strategy, offering a novel insight to the development of NLRP3-targeted therapy.

In summary, this study identified APA regulation as a key role in kidney injury progression, as well as in inflammation overactivation. Additionally, this study offered FIP1 as a promising therapeutic target for kidney injury.

\section{Acknowledgements}

We sincerely thank Dr Liutao Chen for providing us with HK2 cells.

\section{Author contributions}

N.N. and Y.T. designed the experiments. T.Z. and Y.T. performed the experiments. T.Z. and Y.T. wrote the manuscript. N.N., T.Z., and Y.T. supervised the manuscript. J.Q. provided the CKD specimens. X.H., J.Z., and Z.X. provided instructions and help in animal experiments. All authors approved the final version of the manuscript.

\section{Funding}

This research was supported by the National Natural Science Foundation of China (No.82000715, No. 81470977, and No. 81970652); Guangdong Basic and Applied Basic Research Foundation (No. 2019A1515011219); and The Science and Technology Planning Project of Guangzhou (No. 201803010016).

\section{Ethical approval}

This study was approved by the Ethics Committee of The Third Affiliated Hospital of Sun Yat-sen University. The animal experiments were approved by the Institutional Animal Care and Use of The Third Affiliated Hospital of Sun Yat-Sen University.

\section{Conflict of interest}

The authors declare no competing interests.

\section{Publisher's note}

Springer Nature remains neutral with regard to jurisdictional claims in published maps and institutional affiliations.

Supplementary information The online version contains supplementary material available at https://doi.org/10.1038/s41419-021-03751-3.

Received: 11 November 2020 Revised: 20 April 2021 Accepted: 21 April 2021

Published online: 19 May 2021

\section{References}

1. Liu, H. et al. Inhibition of Brd4 alleviates renal ischemia/reperfusion injuryinduced apoptosis and endoplasmic reticulum stress by blocking FoxO4mediated oxidative stress. Redox Biol. 24, 101195 (2019).

2. Lu, Q. B. et al. Salusin-beta mediates tubular cell apoptosis in acute kidney injury: Involvement of the PKC/ROS signaling pathway. Redox Biol. 30, 101411 (2020).

3. Kramann, R. et al. Pharmacological GLI2 inhibition prevents myofibroblast cellcycle progression and reduces kidney fibrosis. J. Clin. Invest. 125, 2935-2951 (2015).

4. Tan, R. Z. et al. BAY61-3606 protects kidney from acute ischemia/reperfusion injury through inhibiting spleen tyrosine kinase and suppressing inflammatory macrophage response. FASEB J. 34, 15029-15046 (2020).

5. Ratliff, B. B., Abdulmahdi, W., Pawar, R. \& Wolin, M. S. Oxidant mechanisms in renal injury and disease. Antioxid. Redox Signal. 25, 119-146 (2016).

6. Tucker, P. S., Scanlan, A. T. \& Dalbo, V. J. Chronic kidney disease influences multiple systems: describing the relationship between oxidative stress, inflammation, kidney damage, and concomitant disease. Oxid. Med. Cell. Longev. 2015, 806358 (2015)

7. Kim Y. G., Kim S. M., Kim K. P., Lee S. H., Moon J. Y. The role of inflammasomedependent and inflammasome-independent NLRP3 in the Kidney. Cells 8 1389 (2019).

8. Andrade-Oliveira, V., Foresto-Neto, O., Watanabe, I. K. M., Zatz, R. \& Camara, N. O. S. Inflammation in renal diseases: new and old players. Front. Pharmacol. 10, 1192 (2019).

9. Tang, P. M. et al. Neural transcription factor Pou4f1 promotes renal fibrosis via macrophage-myofibroblast transition. Proc. Natl Acad. Sci. USA 117, 20741-20752. (2020).

10. Hong, J. et al. Lysosomal regulation of extracellular vesicle excretion during dribose-induced NLRP3 inflammasome activation in podocytes. Biochim. Biophys. Acta Mol. Cell Res. 1866, 849-860 (2019).

11. Vilaysane, A. et al. The NLRP3 inflammasome promotes renal inflammation and contributes to CKD. J. Am. Soc. Nephrol. 21, 1732-1744 (2010).

12. Komada, T. \& Muruve, D. A. The role of inflammasomes in kidney disease. Nat. Rev. Nephrol. 15, 501-520 (2019). 
13. Lorenz, G., Darisipudi, M. N. \& Anders, H. J. Canonical and non-canonical effects of the NLRP3 inflammasome in kidney inflammation and fibrosis. Nephrol. Dial. Transplant 29, 41-48 (2014).

14. Jiang, $\mathrm{H}$. et al. Identification of a selective and direct NLRP3 inhibitor to treat inflammatory disorders. J. Exp. Med. 214, 3219-38. (2017).

15. Kataoka, K. et al. Aberrant PD-L1 expression through 3'-UTR disruption in multiple cancers. Nature 534, 402-406 (2016).

16. Tian, B. \& Manley, J. L. Alternative polyadenylation of mRNA precursors. Nat. Rev. Mol. Cell Biol. 18, 18-30 (2017).

17. Elkon, R., Ugalde, A. P. \& Agami, R. Alternative cleavage and polyadenylation: extent, regulation and function. Nat. Rev. Genet. 14, 496-506 (2013).

18. Cong, J., Gong, J., Yang, C., Xia, Z. \& Zhang, H. miR-22 suppresses tumor invasion and metastasis in colorectal cancer by targeting NLRP3. Cancer Manag. Res. 12, 5419-5429 (2020).

19. Wang, D. et al. MicroRNA-223 negatively regulates LPS-induced inflammatory responses by targeting NLRP3 in human dental pulp fibroblasts. Int. Endod. J. 54, 241-254 (2021).

20. Xu, D., Zhang, X., Chen, X., Yang, S. \& Chen, H. Inhibition of miR-223 attenuates the NLRP3 inflammasome activation, fibrosis, and apoptosis in diabetic cardiomyopathy. Life Sci. 256, 117980 (2020).

21. Zheng, T., Lu, M., Wang, T., Zhang, C. \& Du, X. NRBE3 promotes metastasis of breast cancer by down-regulating E-cadherin expression. Biochim Biophys. Acta Mol. Cell Res. 1865, 1869-1877 (2018).

22. Huang, J. et al. Suppression of cleavage factor Im 25 promotes the proliferation of lung cancer cells through alternative polyadenylation. Biochem. Biophys. Res. Commun. 503, 856-862 (2018).

23. Chen, $X$. et al. CSTF2-induced shortening of the RAC1 3'UTR promotes the pathogenesis of urothelial carcinoma of the bladder. Cancer Res. 78, 5848-5862 (2018).

24. Liu, X. Y. et al. Renal inhibition of miR-181a ameliorates 5-fluorouracil-induced mesangial cell apoptosis and nephrotoxicity. Cell Death Dis. 9, 610 (2018).
25. Chi, H. H. et al. IL-36 signaling facilitates activation of the NLRP3 inflammasome and IL-23/LL-17 axis in renal inflammation and fibrosis. J. Am. Soc. Nephrol. 28, 2022-2037 (2017).

26. Mulay, S. R. Multifactorial functions of the inflammasome component NLRP3 in pathogenesis of chronic kidney diseases. Kidney Int. 96, 58-66 (2019).

27. Wang, W. et al. Inflammasome-independent NLRP3 augments TGF-beta signaling in kidney epithelium. J. Immunol. 190, 1239-1249 (2013).

28. Thivierge, $C$. et al. Alternative polyadenylation confers Pten mRNAs stability and resistance to microRNAs. Nucleic Acids Res. 46, 10340-10352 (2018).

29. Lackford, B. et al. Fip1 regulates mRNA alternative polyadenylation to promote stem cell self-renewal. EMBO J. 33, 878-889 (2014).

30. Park, S. M. et al. U2AF35(S34F) promotes transformation by directing aberrant ATG7 Pre-mRNA 3' end formation. Mol. Cell 62, 479-490 (2016).

31. Kaufmann, I., Martin, G., Friedlein, A., Langen, H. \& Keller, W. Human Fip1 is a subunit of CPSF that binds to U-rich RNA elements and stimulates poly(A) polymerase. EMBO J. 23, 616-626 (2004).

32. Richter, K., Konzack, A., Pihlajaniemi, T., Heljasvaara, R. \& Kietzmann, T. Redoxfibrosis: Impact of TGFbeta1 on ROS generators, mediators and functional consequences. Redox Biol. 6, 344-352 (2015).

33. Bauernfeind, F. et al. Cutting edge: reactive oxygen species inhibitors block priming, but not activation, of the NLRP3 inflammasome. J. Immunol. 187, 613-617 (2011).

34. Zhao, W. et al. AKT regulates NLRP3 inflammasome activation by phosphorylating NLRP3 serine 5. J. Immunol. 205, 2255-2264 (2020).

35. Zheng, S. et al. Posttreatment of Maresin1 Inhibits NLRP3 inflammasome activation via promotion of NLRP3 ubiquitination. FASEB J. 34, 11944-11956 (2020).

36. Zhou, L. et al. Excessive deubiquitination of NLRP3-R779C variant contributes to very-early-onset inflammatory bowel disease development. J. Allergy Clin. Immunol. 147, 267-279 (2021). 